\title{
A Trust-Enabling Support for Goal-Based Services
}

\author{
Luiz Olavo Bonino da Silva Santos, Luis Ferreira Pires, and Marten van Sinderen \\ Centre for Telematics and Information Technology, University of Twente \\ P.O. Box 217, 7500AE, Enschede, The Netherlands \\ E-mail: $\{$ l.o.bonino, l.ferreirapires,m..j.vansinderen\}@ewi.utwente.nl
}

\begin{abstract}
Service-Oriented Computing allows new applications to be developed by using and/or combining services offered by different providers. In several cases a service needs sensitive information from the clients in order to execute. The existence of a trust relationship between the client and the provider determines which restrictions the service has concerning the use of this information by the service. In this paper we present a metamodel for services computing that incorporates the support for trust relationships. The metamodel provides the means for specifying services and service requests including trust requirements and constraints. The proposed metamodel is encompassed in a framework for goal-based service discovery and composition.
\end{abstract}

\section{Introduction}

Service-Oriented Computing (SOC) has emerged as a computing paradigm that uses the concept of service as the basic construct for distributed applications. SOC has a promising vision of a world of cooperating services where cooperation relations can be dynamically created to form applications and business processes [5]. In a simple SOC setting we have a service client that requests the execution of a service to a service provider. Typically, a service needs to gather information from the service client as the input data for its execution. In case of a single request and if only one service provider is considered, it is straightforward for the service client to decide whether the required information is sensitive or not and whether it can be sent to the service. Even if the information is sensitive, in this simple scenario the service client can decide in an ad hoc manner to send it to the service if a (implicit or explicit) trust relationship exists between the service client and the service provider.

However, in environments where a large number of service clients and service providers are present, it becomes difficult to manually and individually assess the sensitiveness of the information as well as control and manage the trust relationships between service clients and service providers. In such environments, the need for a supporting service platform emerges. A service platform can support service clients in activities such as service discovery, selection, composition and invocation [2]. The support can also be extended to service providers by providing a mechanism for rapid creation, deployment and advertisement of services. The addition of semantics to service descriptions and to messages exchanged between service clients, service providers and the supporting service platform enables complex reasoning tasks [8]. Among these reasoning tasks are the interpretation of service providers' capabilities and service clients' requirements.

Consider initially a scenario where the interactions between service clients, service providers and the supporting platform are solely based on the syntax of the exchange messages. In this scenario, activities such as service discovery can be performed by matching the terms contained in the request of a service client with the terms in the description of a service. The terms should have an exact match. This implies that problems can arise if for instance the service client maps concept $C$ to term $T 1$ and the service provider maps this same concept $C$ to term $T 2$. This semantic mismatch can be solved or at least minimized by the introduction of semantic annotations. Assuming that the participants share the same conceptual model and that the terms in the exchanged messages are mapped to this model, a semantic interoperability becomes possible.

In this work, we propose to raise the abstraction level of interoperability. At the higher abstraction level, the organizational and social levels are considered and the mapping is established between a client's goal and the service that fulfills that goal. This paper presents a framework for goalbased dynamic service discovery and composition. The framework includes a precise definition of goal, techniques for goal assessment and modeling, and the rationale for dynamic service discovery and composition. Trust aspects are included in all components of the framework. The approach to tackle trust issues is the focus of this paper.

This document is further structured as follows. Section 
2 presents our goal-based service framework. Section 3 details the proposed metamodel focusing on the trust aspects. Section 4 presents a usage scenario to illustrate the applicability of the framework, and Section 5 gives conclusions and identifies topics for future work.

\section{The goal-based service framework}

In recent years, goal-based analysis has been used in different areas of Computer Science to identify stakeholders' objectives, determine requirements for software systems and guide system's behavior. As a representation of a user's objectives, goals are used in Service-Oriented Computing to indicate the desired outcome of a service. In the Service-Oriented Computing literature we can find initiatives for service discovery and composition based on goals such as the Web Service Modeling Ontology (WSMO) [1], GoalMorph [9] and the approach presented in [10]. These initiatives have in common the assumption that goal definitions are already available and have been previously identified and modeled. However, these initiatives do not clarify how these goals are gathered and modeled and how the goal descriptions relate to concrete services. Moreover, trustrelated issues are treated ad-hoc and mainly by passing to the service client the responsibility to decide to whom information should be provided. We claim that prescriptions of how to model goals and how to define trust relationships are still missing and that they should be addressed when developing a dynamic service discovery and composition framework based on goal gathering and modeling.

Our framework to support dynamic service discovery and composition is based on goal modeling, and assumes that the involved stakeholders (client applications, service providers, supporting platform) are placed in a common and known domain. This requirement is necessary because the approach relies on the availability of domain-specific ontologies. For each domain, valid goals for the stakeholders of that domain are identified together with the tasks required for their fulfillment. Moreover, trust relationships can be defined at the modeling level allowing the supporting platform to enforce these relationships. Figure 1 depicts the main elements that comprise our framework:

- Service platform. A service platform supports the interaction between service providers and service clients. From the service provider's perspective, the platform supports the publication of service descriptions. From the service client's perspective, the platform provides facilities for service discovery, composition, invocation and monitoring, among others. Different platform implementations can offer different sets of facilities. In this work we assume that the platform offers to the service client at least service discovery and composition.
- Goal-based service metamodel. This metamodel defines domain-independent concepts such as service, stakeholder, organization, goal and task, and their relations. These definitions are further used and specialized in the domain and task ontologies.

- Domain ontologies. These ontologies define domainspecific concepts and the relations among these concepts. Domain ontologies should be available for the service platform as well as for the service providers and clients.

- Task ontologies. Associated with domain ontologies, task ontologies provide domain-specific definitions of the tasks valid in a domain. These task definitions include not only a list of valid tasks in a domain but also the structure of these tasks, i.e., dependency relations among tasks and task decomposition.

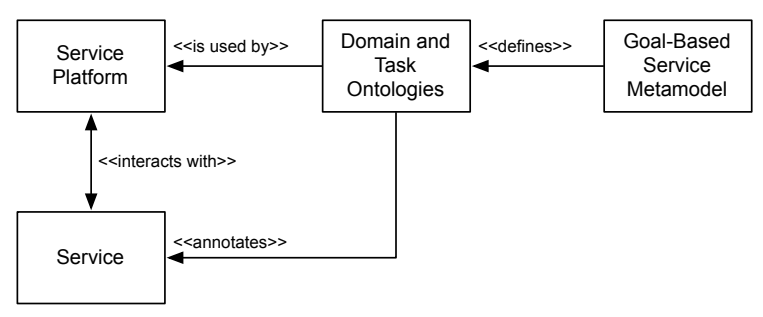

\section{Figure 1. Main components of the goal-based services framework}

The concept of goal has several different definitions varying from "the result of scoring"and "the physical structure that defines where the score is achieved" in some ball games to "a statement of intent for the direction of the business" in business administration. The definitions strongly depend on to which application area this term is applied. Narrowing down to the Computer Science domain, a variety of definitions of the goal concept can also be found. In the Artificial Intelligence (AI) realm, goal is defined as a "description of a world state that is expected to be realized" [7]. Among the several definitions for goal in the agent-oriented computing community in [4] goal is defined as a "state with highest utility and an agent must choose the course of action to reach that goal" and in [6] goal is defined as a "final state that the agent tries to achieve by moving from its initial state through a defined and finite sequence of intermediary states".

For the purposes of this framework, we define goal as $a$ description of an agent's intended state of affairs. This definition encompasses two aspects: $(i)$ the agent has a setting of the world that he/she wants to be reached, and (ii) the agent is committed to the fulfillment of the goal and will act 
accordingly to have it fulfilled. The latter aspect is also referred to as the agent's intentionality. A task is defined here as the means to fulfill a goal, i.e., it defines a behavior that transforms the world from a setting $A$ into a setting $B$. When the state of affairs derived by task $T$ 's outcome matches the description of the state of affairs of goal $G$, we say that task $T$ supports goal $G$.

Figure 2 depicts the relations between goals, tasks and the related agents. In Figure 2 we derive two other relations:

1. If $A g e n t \_B$ executes $T a s k \_A$ and Task $A$ supports Goal_A, it implies that Goal_A is fulfilled by Agent_B.

2. If $A g e n t \_A$ has Goal $A$ and, for some reason it cannot fulfill the goal itself, a delegation is performed. This delegation implies trust relationships and commitments that are further detailed in next sections.

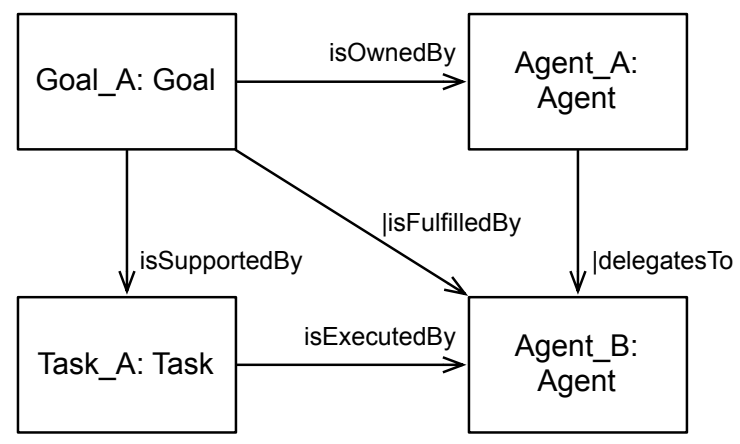

Figure 2. Relations between goals and tasks

\section{The service metamodel}

We related our definition of goal and task from Section 2 with concepts of the Service-Oriented Computing realm. The notion of agent, as used in Figure 2 is refined by the notion of service client and service provider. Figure 3 depicts the relations between goals, tasks, services, service clients and service providers.

In our approach we consider a service the concrete realization of a task. In other words, a task is an abstract (in the sense that it does not have a direct implementation) definition of activities whose outcome can match the state of affairs defined by a goal. This separation between a definition of activities and the actual implementation of these activities allows the division of administrative domains of tasks and services. While services are typically defined and operated by service providers, tasks can be defined not only by service providers but also by the service client, domain specialists or by the providers of the supporting service platform. In the scope of our work as depicted in Figure 1,

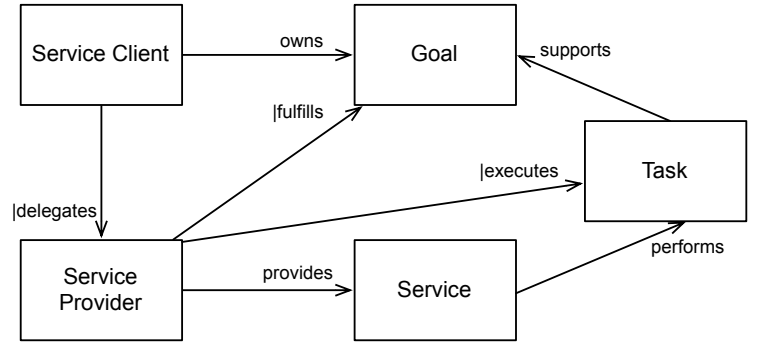

Figure 3. Relations between goals, tasks and services

we assume that task definition is mainly done by domain specialists by means of task ontologies associated with domain ontologies. In this scenario the following steps are followed:

1. Domain specialists define domain and task ontologies including goals valid in a domain;

2. Service providers define their services and make them available to the supporting service platform. These services are semantically annotated according to the available domain ontologies;

3. The supporting service platform tries to match the services with the tasks defined in the task ontologies;

4. Service clients define their goals and request the fulfillments of these goals to the supporting service platform;

5. The supporting service platform matches clientdefined goals with the domain ontology goals.

After these steps, we have a relation between a clientdefined goal, its counterpart domain-defined goal, a task supporting the goal and a service that performs the task. Once this relation is established, a service can be executed and its outcome fulfills the service client's goal.

These steps are only possible when a trust relationship exists between service client and service providers. This relationship can be direct, i.e., a service client A trusts a service provider B; or it can be indirect (by transitivity), i.e., the service client A trusts the supporting service platform and, indirectly, trusts the service providers associated with the service platform. In these two scenarios we have binary relationships relating service clients to service providers or to the service platform. However, more complex scenarios emerge when we consider that the trust relationship has a context. Considering the real-world example where John trusts Peter to borrow his car but does not trust him his personal account's password. In this example, the trust 
relationship includes a conditional element (borrowing of John's car) that limits its existence.

In our framework, the conditional element of a trust relationship is the goal. By including a goal in the trust relationship between a service provider and a service client we define trust as a ternary relationship as depicted in Figure 4. The trust, ownership and delegation relations in our framework are based on and extend the relations presented in the Secure Tropos modeling framework [3] and are defined as follows:

- Ownership. Represents the fact that the service client is the legitimate owner of the goal. The owner has full authority over the owned goal and can grant its fulfillment to another agent (the service platform or a service provider);

- Trust. Represents the belief of one agent (the service client) that another agent (the service platform or a service provider) will not misuse the goal it has been granted. The former actor is called truster, the later is called trustee and the object around which the trust is centered is called trustum;

- Delegation. Represents a formal event on which an agent delegates to another agent the permission to fulfill a goal. The former agent is called delegater, the later agent is called delegatee and the object of the delegation is called delegatum.

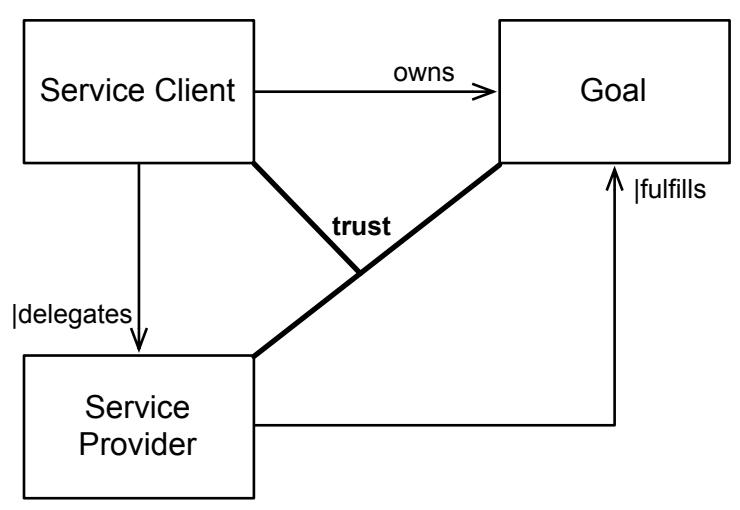

Figure 4. Trust relationship

Regarding trust, the role of the supporting platform is to only allow the establishment of the delegation of a service client's goal to a service provider if a trust relationship exists between them. Ultimately, the service platform intermediates the discovery and selection of services according to the client's goals. Therefore, the delegation relationship is complete only when the service platform discovers, selects and invokes a service that matches the requirements of the client's goal and a trust relationship can be established (directly or indirectly) between the service client and the service provider.

The trust relationship can be indicated in two alternative ways: $(i)$ the service client explicitly informs to the supporting platform the service providers that it trusts or; (ii) the service client informs to the supporting platform the conditions for trusted service providers. In the former case, the platform only selects services from providers that have trust relationships with the requesting service client. In the later case, the service platform receives the conditions from the client and matches these conditions to the information concerning the providers. For instance, a service client can define that it only trusts providers that are located in its city. In this example the service platform only selects the services from providers located in the same city of the service client.

\section{Example scenario}

To illustrate the applicability of our framework, we chose the Domotics domain for our example scenario. In the example, John (a service client) has a goal of getting customized comfort settings whenever he arrives at home. Figure 5 depicts the ownership relation between a service client (John) and its goal (GetCustomizedComfort). This relationship is automatically assessed by the supporting platform when the service client informs the platform the goal it wants to be fulfilled.

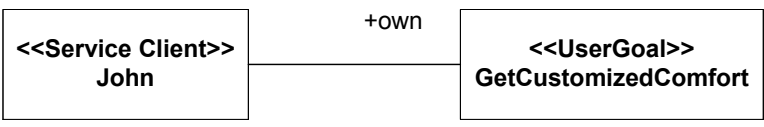

Figure 5. Service client's goal

Among the several goals defined in the domotics domain ontology, in this paper we consider one to customize the comfort settings of the house according to the user. This goal specifies which comfort settings the user wants, e.g., ambience lighting, temperature, etc. Figure 6 shows the model defined by domain specialists in which the domaindefined goal GetCustomizedComfort is supported by the task SetLights. In this model, a task decomposition structure is also presented. The model shows that the task SetLights can be composed by the sub-tasks SetLightColor and SetLightIntensity. This decomposition model allows the platform to search for services that can perform the activities defined in the SetLights task. If no service could be found to perform the activities of the SetLights task, the platform searches for services that can perform the activities defined in the sub-tasks SetLightColor and SetLightIntensity. 


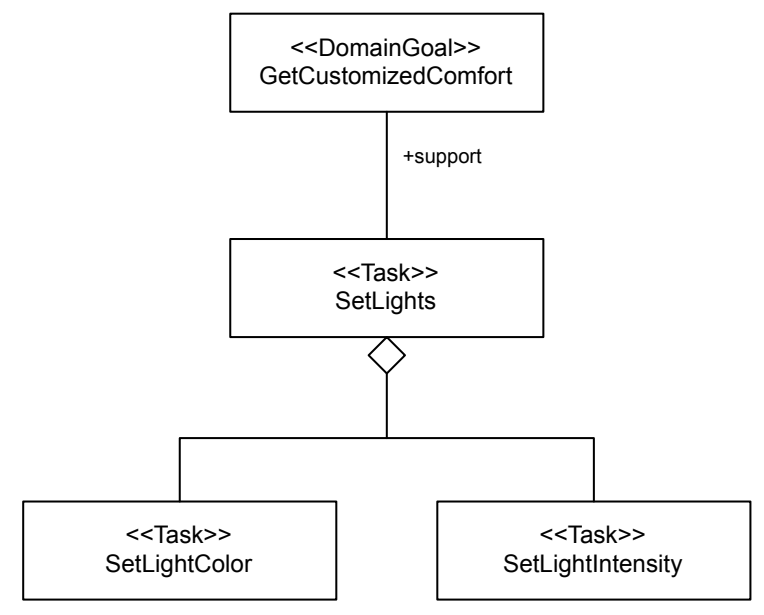

Figure 6. Domain goal and tasks

Figure 7 shows the trust relationships defined by the service client and informed to the service platform. In this example, John defines that he trusts the service providers ServProv $\_$A and ServProv_B. Based on this information the service platform proceeds to search for services provided by the service providers trusted by John and that perform the activities defined in the tasks depicted in Figure 6.

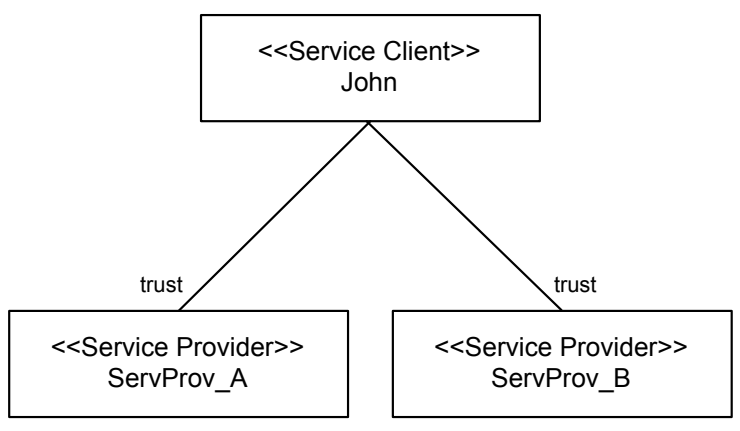

Figure 7. Service client's trust relationships

Figure 8 shows the services provided by three service providers. If trust is not considered, the first choice of service according to the goal and task model presented in Figure 6 would be the SetLights service provided by the ServProv_C service provider. However, as depicted in Figure 7 John does not have a trust relationship with the ServProv_C service provider. Therefore, the platform cannot choose services provided by this service provider for the client John. Since no service has been found that performs the activities defined in the SetLights task, the platform proceeds to the sub-tasks defined on the task decomposition structure. In this case, the platform finds sub-tasks that com- pose the SetLights task and searches for services performing the activities defined in these sub-tasks from trusted service providers.
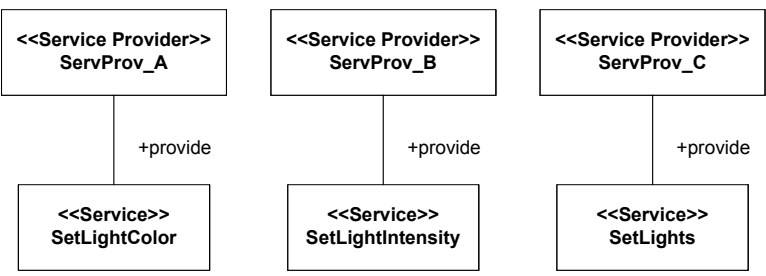

\section{Figure 8. Service providers and their services}

The platform finds the services SetLightColor and SetLightIntensity that performs the activities defined in subtasks SetLightColor and SetLightIntensity, respectively. Now the supporting platform could establish a relation between the client's goal (GetCustomizedComfort), its related domain goal (GetCustomizedComfort), the task that supports the domain goal (SetLights), its sub-tasks (SetLightColor and SetLightIntensity) and the services that perform these sub-tasks (SetLightColor and SetLightIntensity). Having established this relation, the platform succeeds in finding the services that fulfill the client's goal.

\section{Conclusions and future work}

In this paper we presented a metamodel for goal-based semantic services that enables the definition and enforcement of trust relationships. This metamodel is part of a framework that supports goal-based semantic services. Besides the metamodel, the framework is composed by domain and task ontologies and a service supporting platform.

The metamodel defines goals as the description of a service client's intended state of affairs. The domain and task ontologies define valid goals for a specific domain and tasks that support the fulfillment of these goals. Finally, services are offered to the platform by service providers and are also annotated according to the domain ontologies. When the fulfillment of a goal is requested by a service client, the supporting platform matches the goal to the domain-defined goals and retrieves the tasks defined on the task ontologies that support the goal. A task is an abstract description of activities that can, as its outcome, fulfill a goal. The service is defined here as the concrete realization of activities prescribed by a service provider.

The distinction between the abstract description of activities (the task) and the concrete realization of activities (the service) has been shown useful in our framework to support dynamic service discovery.

The framework assumes the previous existence of domain and task ontologies defined by domain specialists. 
This assumption makes the framework suitable for environments where the domain is clear and well known. If the domain changes, the domain and task ontologies should be updated to reflect the new conditions.

The mechanism for trust enforcement is embedded in the goal-based service metamodel and starts with the service client defining a list of service providers it trusts or defining criteria for potential trusted service providers. Then the supporting service platform searches for services from trusted service providers or for service providers that comply with the informed trust criteria. Preliminary evaluation shows that the definition of trust criteria offers more flexibility. In this way the service client does not need to inform the platform every time it trusts a new service provider. The platform uses the trust criteria to automatically infer whether a service provider can be trusted by the service client or not.

The next steps of our research on this trust-enabling and enforcement framework are: (i) the definition of more complex domain and tasks ontologies; (ii) the complete implementation of the supporting platform; (iii) the definition of evaluation criteria for the framework and;(iv) the comprehensive evaluation of the framework against the defined criteria.

\section{Acknowledgements}

The present work is partly funded by the Freeband Communication project A-Muse (http://a-muse.freeband.nl). AMuse is sponsored by the Dutch government under contract BSIK 03025. The work on the definition of the goal concept is being conducted in cooperation with Dr. Giancarlo Guizzardi, Universidade Federal do Esprito Santo, Brazil.

\section{References}

[1] S. Arroyo, E. Cimpian, J. Domingue, C. Feier, D. Fensel, B. Knig-Ries, H. Lausen, A. Polleres, and M. Stollberg. Web service modeling ontology primer. W3C Member Submission, June 2005.

[2] L. O. Bonino da Silva Santos, M. van Sinderen, and L. Ferreira Pires. Architectural models for client interaction on service-oriented platforms. In M. van Sinderen, editor, 1st International Workshop on Architectures, Concepts and Technologies for Service Oriented Computing (ACT4SOC 2007), pages 19-27. INSTIC, July 2007.

[3] P. Giorgini, F. Massacci, J. Mylopoulous, and N. Zannone. Requirements engineering meets trust management: Model, methodology, and reasoning. In In Proceedings of the 2th International Conference on Trust Management, April 2004.

[4] M. Moghadasi, A. Haghighat, and S. Ghidary. Evaluating markov decision process as a model for decision making under uncertainty environment. Machine Learning and $C y$ bernetics, 2007 International Conference on, 5:2446-2450, Aug. 2007.
[5] M. P. Papazoglou, P. Traverso, S. Dustdar, and F. Leymann. Service-oriented computing research roadmap. Technical report, European Union Information Society Technologies (IST), Directorate D, 2006.

[6] J. S. Rosenschein and G. Zlotkin. Rules of encounter: designing conventions for automated negotiation among computers. MIT Press, Cambridge, MA, USA, 1994.

[7] S. J. Russell and P. Norvig. Artificial Intelligence: A Modern Approach. Pearson Education, 2003.

[8] K. Sycara, M. Paolucci, J. Soudry, and N. Srinivasan. Dynamic discovery and coordination of agent-based semantic web services. IEEE Internet Computing, 8(3):66-73, 2004.

[9] M. Vukovic and P. Robinson. Goalmorph: partial goal satisfaction for flexible service composition. In Proc. International Conference on Next Generation Web Services Practices NWeSP 2005, page 6pp., 22-26 Aug. 2005.

[10] K. Zhang, Q. Li, and Q. Sui. A goal-driven approach of service composition for pervasive computing. In Proc. 1st International Symposium on Pervasive Computing and Applications, pages 593-598, 3-5 Aug. 2006. 